Structure learning using a focused information criterion in graphical models

Eugen Pircalabelu, Gerda Claeskens, and Lourens Waldorp

DEPARTMENT OF DECISION SCIENCES AND INFORMATION MANAGEMENT (KBI) 


\title{
Structure Learning using a Focused Information Criterion in Graphical Models
}

Eugen Pircalabelu
Gerda Claeskens

ORSTAT and Leuven Statistics Research Center

KU Leuven

Naamsestraat 69, 3000 Leuven, Belgium

Lourens Waldorp

Department of Psychological Methods

University of Amsterdam

Weesperplein 4, 1018 XA Amsterdam, the Netherlands
EUGEN.PIRCALABELU@KULEUVEN.BE GERDA.CLAESKENS@KULEUVEN.BE

WALDORP@UVA.NL

\begin{abstract}
A new method for model selection for Gaussian directed acyclic graphs (DAG) and Gaussian graphical models (GGM), with extensions towards ancestral graphs (AG), is constructed to have good prediction properties. The method is based on the focused information criterion, and offers the possibility of fitting individual tailored models. The focus of the research, that is, the purpose of the model, directs the selection. It is shown that using the focused information criterion leads to a graph with small mean square error. Two situations that commonly occur in practice are treated: the improvement of an already pre-specified feasible model, and a data-driven full discovery of the graphical structure. The search algorithms are illustrated by means of data examples and are compared with existing methods in a simulation study.
\end{abstract}

Keywords: Focused information criterion; model selection; directed acyclic graph; Gaussian graphical model; ancestral graph.

\section{Introduction}

Probabilistic graphical models are increasingly studied in the statistical and machine learning community, because of their direct applicability to problems coming from areas such as image analysis, engineering, biomedical and computer sciences. The most popular such graphical structures are the directed acyclic graphs (DAG) and Gaussian graphical models (GGM) which have been extensively studied in Whittaker (1990), Lauritzen (1996), Edwards (2000), Koller and Friedman (2009), amongst others.

The main objective of the present paper is to develop a methodology for model selection within candidate models, such that the final selected model has good performance with respect to prediction. For this purpose we use the focused information criterion, FIC (Claeskens and Hjort, 2003). Unlike other information criteria, such as the traditional Akaike information criterion (AIC, Akaike, 1973) and the Bayesian information criterion (BIC, Schwarz, 1978), the FIC allows for selecting individual models, tailored to a specific purpose (the focus), as opposed to attempting an identification of a single model that should be used for all purposes. The proposed search algorithms fall into the 'score-based' model selection category, where models receive a corresponding score, in this case based on an 
estimated mean squared error (MSE) value of the focus estimator. While the FIC has been applied to generalized linear models (Claeskens and Hjort, 2008b), in models for survival data (Hjort and Claeskens, 2006), in generalized partial linear models (Zhang and Liang, 2011), and in several other types of models. Its definition, computation and application to graphical models is new. The advantages of using such an estimated model are two-fold: first, if certain assumptions hold (see Section 3), the estimated graph will provide low MSE for the selected focus estimator, and second, given a particular configuration in the covariate space (e.g., for a given individual), a model for such a configuration can be selected. Selecting one global model, though still having low MSE for the focus estimator, is another possibility of the focused selection.

\section{Notation for Graphical Models}

We denote by $\mathcal{G}(\mathcal{E}, \mathcal{V})$ a graphical structure (or graph) based on a set of nodes $(\mathcal{V})$, a set of edges $(\mathcal{E})$, and a set of random variables $\left\{X_{i}: i \in \mathcal{V}\right\}$. For $\mathcal{V}=\{1, \ldots, p\}$, each of the variables $X_{1}, \ldots, X_{p}$ corresponds to one node (or vertex) in the set $\mathcal{V}$. The set of edges (or arcs) $\mathcal{E}$ is a subset of $\mathcal{V} \times \mathcal{V}$, the set of ordered pairs of distinct nodes. A connection between two nodes (say, $i$ and $j$ ) can be either undirected $(i-j)$ or directed $(i \rightarrow j$ or $i \leftarrow j$ ). We denote a directed edge $j \leftarrow i$ in $\mathcal{E}$ by $(j, i)$. We call node $i$ (or variable $X_{i}$ ) a parent of node $j$ (or variable $X_{j}$ ), conversely node $j$ is referred to as a child of node $i$. To make the notation easier, an undirected edge $i-j$ is set between nodes $i$ and $j$ if and only if $\mathcal{E}$ contains both $(i, j)$ and $(j, i)$, and call $i$ and $j$ adjacent (or neighbors). A directed path between nodes $i$ and $z$ is a sequence of nodes that starts in $i$ and by following the directionality of the arrows leads to node $z$ (e.g. $i \rightarrow j \rightarrow \ldots \rightarrow y \rightarrow z$ ). Node $i$ is referred to as an ancestor of $z$ if there exists such a directed path between the two nodes, or if $i=z$. For later use, we define a third type of arrow $i \leftrightarrow j$ which will be used to refer two nodes as being 'spouses'. Only one connection can be made between two nodes $i$ and $j$, be it directed, undirected or bidirected, and no self-loop edges are permitted (such as $i \rightarrow i, i-i$ or $i \leftrightarrow i$ ).

\subsection{Directed Acyclic Graphs}

Directed acyclic graphs are defined as a class of statistical models, consisting of a graph $\mathcal{G}(\mathcal{E}, \mathcal{V})$ and a probability distribution $P$, with two particular characteristics. First, it contains only directed edges between pairs of vertices, such that there are no feedback loops (referred to as the 'acyclicity' property). That is, any directed path starting at node $i$ cannot lead back to $i$. Second, the joint multivariate probability density function (pdf) of $\left(X_{1}, \ldots, X_{p}\right)$ factorizes with respect to the parents $p a\left(X_{l}\right)$ only, that is,

$$
f\left(x_{1}, \ldots, x_{p}\right)=\prod_{l=1}^{p} f\left(x_{l} \mid p a\left(x_{l}\right)\right),
$$

where the conditioning is on $p a\left(x_{l}\right)$, the set of parental variables of $X_{l}$. Graphically, this is represented by a directed arrow from each of the 'parents' to the 'children'. In the Gaussian DAG (or Gaussian Bayesian net) all conditional pdfs are linear Gaussians (see Koller and Friedman, 2009, chap. $5 \& 7$ ), where $X_{j}$ has a linear Gaussian model if conditional on its 
parents, $X_{1}=x_{1}, \ldots, X_{i}=x_{i}$,

$$
X_{j} \sim N\left(\beta_{j 0}+\beta_{j 1} x_{1}+\ldots+\beta_{j i} x_{i} ; \sigma^{2}\right),
$$

for some values of the regression coefficients $\beta_{j 0}, \ldots, \beta_{j i}$ and of the variance $\sigma^{2}$.

\subsection{Gaussian Graphical Models}

If all edges in $\mathcal{E}$ are undirected, $\mathcal{G}(\mathcal{E}, \mathcal{V})$ is an undirected graph (UG). We assume that $\left(X_{1}, \ldots, X_{p}\right) \sim N_{p}(\mu, \Sigma)$, where without loss of generality we take $\mu=0$. In the literature, this model is known under the name of 'Gaussian graphical model' (GGM), 'concentration graph', or 'covariance selection model' (Dempster, 1972). The concentration matrix $\Sigma^{-1}$ contains information about the covariance between pairs $\left(X_{i}, X_{j}\right)$ conditioned on all other variables in the model.

For the focused model search we use that (see Lauritzen, 1996, chap. 5) if $\left(X_{1}, \ldots, X_{p}\right) \sim$ $N_{p}(0, \Sigma)$, then

$$
(i, j) \&(j, i) \in \mathcal{E} \Leftrightarrow \Sigma_{i j}^{-1} \neq 0 \Leftrightarrow \beta_{i j} \neq 0 \& \beta_{j i} \neq 0,
$$

where the following conditional equation for any particular variable is put forward,

$$
X_{j} \mid\left\{X_{i}: i \in \mathcal{V} \backslash j\right\}=\sum_{i \in \mathcal{V} \backslash j} \beta_{j i} X_{i}+\epsilon_{j}, \quad \epsilon_{j} \sim N\left(0, \Sigma_{j j}\right), \quad \forall j \in \mathcal{V}
$$

Thus an undirected arrow exists between $X_{j}$ and $X_{i}$, if and only if there are non-zero partial regression coefficients in both of the following regression models: regressing $X_{i}$ on $X_{j}$ (given all other nodes) and regressing $X_{j}$ on $X_{i}$ (given all other nodes). The implication of the above reasoning is that the entire covariance estimation problem is translated into regression language (see also Meinshausen and Bühlmann, 2006), which will be exploited in the focused model search.

\subsection{Ancestral Graphs}

Richardson and Spirtes (2002) introduced the 'ancestral graphs' (AG) as a specification of a general 'mixed graph' that can contain three types of edges: undirected $(i-j)$, directed $(i \rightarrow j$ or $i \leftarrow j)$ and bidirected $(i \leftrightarrow j)$. Not all configurations of edges are allowed in an ancestral graph, which is a graph specified by the two conditions that specify that there can be no cycles and no directed edges to nodes with indirected edges, i.e., for all nodes $i$ :

$$
i \notin a n(p a(i) \cup s p(i)) \text { and if } n e(i) \neq \emptyset \text { then } p a(i) \cup s p(i)=\emptyset,
$$

where $a n(i), p a(i), s p(i), n e(i)$ are the corresponding sets of ancestors, parents, spouses and neighbors of node $i$.

Under the assumption of joint multivariate normality for the variables of interest, Drton and Richardson (2004) have provided an algorithm to estimate the covariance matrix $\Sigma$, using the idea of iterative conditional fitting (ICF), where the matrix $\Sigma$ is decomposed as:

$$
\Sigma=(I-B)^{-1}\left(\begin{array}{cc}
\Lambda^{-1} & 0 \\
0 & \Omega
\end{array}\right)\left((I-B)^{-1}\right)^{T} .
$$


The matrices $B, \Lambda$, and $\Omega$ contain non-zero entries corresponding, respectively, to the set of directed $\left(B=\left(\beta_{j i}\right)\right)$, undirected $\left(\Lambda=\left(\lambda_{j i}\right)\right)$ and bidirected $\left(\Omega=\left(\omega_{j i}\right)\right)$ edges. The interpretation of the entries in the three matrices is as follows: $\lambda_{j i}$ represents an inverse covariance element for the subgraph formed by the undirected edges, $-\beta_{j i}$ represents the partial regression coefficient of node $i$ in the relation $j \leftarrow i$ and $\omega_{j i}$ represents the covariance between errors $\epsilon_{i}$ and $\epsilon_{j}$ once the influence of both sets of parents $p a(i)$ and $p a(j)$ has been controlled for, in the regression model $X_{i}=\sum_{l \in p a(i)} \beta_{i l} X_{l}+\epsilon_{i}$ and similarly for $X_{j}$.

Note that the ICF algorithm only performs estimation, rather than learning the structure of the graph (i.e. for a given user prespecified graphical structure, the algorithm estimates the $B, \Lambda, \Omega$ matrices). Spirtes et al. (1999) and Zhang (2008) have proposed a constraintbased approach which using a series of orientation rules, learns from the data a partial ancestral graph (PAG), that belongs to the Markov equivalence class of an AG. It is a partial $\mathrm{AG}$, in the sense that, there are possibly six kinds of edges $-, \rightarrow, \leftrightarrow, \circ-, \circ-\circ, \circ \longrightarrow$, as the extra $\circ$ symbol denotes an undetermined edge mark.

\section{FIC for Model Selection in Graphical Models}

Consider a dataset consisting of $n$ independent cases for each $p$-dimensional vector $\left(X_{k 1}, \ldots\right.$, $X_{k p}$ ) where for each $k=1, \ldots, n$ we assume a linear Gaussian model such that for each fixed $i \in \mathcal{V}$ the variables $\left\{X_{k i: k=1, \ldots, n}\right\}$ are independent. That is, conditional on $\left\{X_{k i}: i \in \mathcal{V} \backslash j\right\}$,

$$
X_{k j} \sim N\left(\sum_{i \in \mathcal{V} \backslash j} \beta_{j i} X_{k i}, \Sigma_{j j}\right), \forall j \in \mathcal{V} \& \forall k=1, \ldots, n
$$

To facilitate model selection, for each $j \in \mathcal{V}$ we introduce a $p-1$ dimensional vector $\gamma_{j}$ with $i$ th element $(i \in \mathcal{V})$

$$
\gamma_{j i}= \begin{cases}\beta_{j i} & \text { if } X_{i} \text { is a parent of } X_{j} \\ 0 & \text { otherwise. }\end{cases}
$$

We define the vector $\theta_{j}$ to contain the parameters that should be estimated in all models and that are never subject to model selection or exclusion. For example, regardless of which parents enter the model, $\Sigma_{j j}$ has to be estimated. If, based on theoretical reasons, a variable $X_{q}$ is decided beforehand to be a parent of $X_{j}$ regardless of what other variables are selected as parents, then also $\beta_{j q}$ is included in $\theta_{j}$.

For each node $j \in \mathcal{V}$, a subset $S \subseteq \mathcal{V} \backslash j$ of possible parents is to be selected. To simplify the notation, we omit the index $j$ since in the remainder of this section all derivations are nodewise. Later, when necessary, the subscript $j$ is reintroduced. There is a one-to-one correspondence between $S$ and $\gamma$. For example, the largest such subset, denoted as $S_{\text {wide }}$ has all $\gamma$ parameters non-zero, while the smallest subset $S_{\text {narrow }}$ has all the $\gamma$ parameters set to zero, corresponding to no parents. For any other $S$ in between the wide and narrow sets, particular elements of the $\gamma$ vector are set to 0 , while others are not. Each model based on $S$ corresponds to working with the density $f\left(X ; \theta, \gamma_{S}\right)$ where particular parameters in the $\gamma$ vector have been set to 0 , according to $S$ (Claeskens and Hjort, 2008a).

Most often, the list of possible parents of variable $X$ is left unspecified, such that an extensive search could be performed. In other situations this list can be constrained beforehand, if knowledge about plausible or implausible relations is available to the researcher. 
For any particular node, we define a focus parameter $\mu=\mu(\theta, \gamma ; x)$ that is a function of the $\theta$ and $\gamma$ parameters of the density, and potentially of a user-specified vector of covariate values $x$, which is intended to be estimated as precisely (in the MSE sense) as possible (see section 6.1 for an example). In each model based on $S$, a maximum likelihood estimator $\hat{\mu}_{S}=\mu\left(\hat{\theta}_{S}, \hat{\gamma}_{S} ; x\right)$ is constructed. The FIC method estimates $\operatorname{MSE}\left(\hat{\mu}_{S}\right)$ and selects the model with the smallest such value. Note that the length of the vector $\hat{\theta}_{S}$ is always the same, its value, though, may change with different choices of $S$. Thus for different focuses, different orderings of the MSE values might occur, leading to possibly different selected models, depending on the specific focus or target. In this way, one can obtain better selected models in terms of MSE than obtained from a global model search not taking any use of the selected model (focus) into account. For structure learning in graphs the focus is the expected value of a variable (see Section 4), reflecting the interest in discovering the topology of the graph.

We further introduce the Fisher information matrix (i.e., the expected value of the matrix of second partial derivatives of the log likelihood with respect to the parameters) for model $S, J_{S}=\left(\begin{array}{cc}J_{00, S} & J_{01, S} \\ J_{10, S} & J_{11, S}\end{array}\right)$, (partitioned in blocks according to the dimensions of $\theta$ and $\left.\gamma_{S}\right), Q_{S}=J_{11, S}^{-1}, Q=J_{11}^{-1}$ (with $J$ without subscript the Fisher information matrix for the 'wide' model) and $G_{S}=\pi_{S}^{t} Q_{S} \pi_{S} Q^{-1}$. The projection matrix $\pi_{S}$ with dimension $|S| \times(p-1)$ contains 0 s and 1 s, such that when multiplied with matrices of interest, it retains those rows and columns that relate to the parameters contained in model $S$, e.g. for a vector $v$, with $\pi_{\{2\}}=(0,1,0, \ldots, 0), \pi_{\{2\}} v=v_{2}$, the second component of $v$ (see Claeskens and Hjort, 2008a, p. 146).

To balance the contributions of the squared bias and variance of the estimators $\hat{\mu}_{S}$, similarly as in Hjort and Claeskens (2003), we consider a local misspecification setting, that is, each $X_{k j}$, for $k=1, \ldots, n$, has pdf

$$
f\left(x_{j} \mid p a\left(x_{j}\right) ; \theta_{0}, \gamma_{0}+\delta / \sqrt{n}\right),
$$

where $\theta_{0}$ and $\gamma_{0}$ correspond to the narrow model. Theorem 6.1 in Claeskens and Hjort (2008a) asserts that under certain conditions the maximum likelihood estimator of the focus parameter obeys

$$
\sqrt{n}\left(\hat{\mu}_{S}-\mu_{\text {true }}\right) \stackrel{d}{\rightarrow} \Lambda_{0}+\omega^{t}\left(\delta-G_{S} D\right),
$$

where $\Lambda_{0} \sim N\left(0,\left(\frac{\partial \mu}{\partial \theta}\right)^{t} J_{00}^{-1} \frac{\partial \mu}{\partial \theta}\right), \omega=J_{10} J_{00}^{-1} \frac{\partial \mu}{\partial \theta}-\frac{\partial \mu}{\partial \gamma}, D \sim N(\delta, Q)$ and $\mu_{\text {true }}=\mu\left(\theta_{0}, \gamma_{0}+\right.$ $\delta / \sqrt{n})$.

Based on the quantities defined above, and defining $I_{p-1}$ as the $(p-1) \times(p-1)$ identity matrix, the limiting expression for $\operatorname{MSE}\left(\hat{\mu}_{S}\right)$ is

$$
\operatorname{MSE}\left(\hat{\mu}_{S}\right)=\left(\frac{\partial \mu}{\partial \theta}\right)^{t} J_{00}^{-1} \frac{\partial \mu}{\partial \theta}+\omega^{t} \pi_{S}^{t} Q_{S} \pi_{S} Q^{-1} \omega+\omega^{t}\left(I_{p-1}-G_{S}\right) \delta \delta^{t}\left(I_{p-1}-G_{S}\right)^{t} \omega .
$$

An asymptotically unbiased estimator is obtained by plugging-in the sample version of the unknown quantities, and evaluating $\hat{J}, \frac{\partial \hat{\mu}}{\partial \theta}$ and $\frac{\partial \hat{\mu}}{\partial \gamma}$ using estimates in the wide model, with $\hat{\delta}=\sqrt{n} \hat{\gamma}$. This leads to the estimated MSE,

$$
\widehat{\operatorname{MSE}}\left(\hat{\mu}_{S}\right)=\left(\frac{\partial \mu}{\partial \theta}\right)^{t} \hat{J}_{00}^{-1} \frac{\partial \mu}{\partial \theta}+2 \hat{\omega}^{t} \pi_{S}^{t} \hat{Q}_{S} \pi_{S} Q^{-1} \hat{\omega}+\hat{\omega}^{t}\left(I_{p-1}-\hat{G}_{S}\right) \hat{\delta} \hat{\delta}^{t}\left(I_{p-1}-\hat{G}_{S}\right)^{t} \hat{\omega}-\hat{\omega}^{t} \hat{Q} \hat{\omega} .
$$


The two terms in the middle of (2) form the estimated focused information criterion, while the remaining two terms, for a given node, are independent of model $S$ and thus independent of which parents are chosen.

Since the limiting and estimated MSE are defined per node, we next define the FIC for the overall estimated graphical structure as the nodewise summation of MSEs, where each node $l \in \mathcal{V}$ has a particular model $S_{l}$ based on which we have estimated $\hat{\mu}_{l ; S_{l}}$,

$$
\operatorname{FIC}\left(\mathcal{G}\left(\mathcal{E}_{\mathcal{S}}, \mathcal{V}\right)\right)=\sum_{l=1}^{p} \widehat{\operatorname{MSE}}\left(\hat{\mu}_{l ; S_{l}}\right),
$$

where $\mathcal{S}=\left\{S_{1}, \ldots, S_{p} \mid S_{1} \subseteq\{\mathcal{V} \backslash 1\} ; \ldots ; S_{p} \subseteq\{\mathcal{V} \backslash p\}\right\}$. The objective is to minimize (3) over $\mathcal{S}$ such that, depending on the context, $\mathcal{G}$ is a DAG, GGM or AG.

Achieving the optimal solution can be performed by complete enumeration followed by testing the selected graphs for DAG, GGM or AG requirements. Since the number of possible combinations of models is of exponential order, this exhaustive procedure can work only for small networks of up to 6 nodes. We propose in Section 4, a faster, 'greedier' approach suitable for larger networks.

Lemma 1, similar to Theorem 3.7 in Williamson (2005, p. 24), justifies theoretically for DAGs the circumstances under which adding an arrow does not increase the KullbackLeibler distance to the target distribution.

Lemma 1 Let the DAGs $\mathcal{G}$ and $\mathcal{H}$ with corresponding positive pdfs $p_{\mathcal{G}}$ and $p_{\mathcal{H}}$ be such that $\mathcal{H}$ differs from $\mathcal{G}$ by only one extra directed arrow between nodes $i$ and $j$, (say, $j \leftarrow$ i). If for a finite set of graphs there exists a unique target distribution $p^{\star}$ that minimizes $\sum_{l=1}^{p} M S E\left(\hat{\mu}_{l ; S_{l}}\right)$, then $p_{\mathcal{H}}$ will be closer to $p^{\star}$ in terms of Kullback-Leiber distance if there exist an uncountable number of points $x_{j}$ such that $f\left(x_{j} \mid x_{i}\right) \neq f\left(x_{j}\right)$ and $X_{i}$ does not equal a point mass at 0 . Otherwise, $p_{\mathcal{H}}$ will not be further away from $p^{\star}$ than $p_{\mathcal{G}}$.

Proof The proof follows closely the one offered in Williamson (2005). Let $d\left(p^{\star}, p_{\mathcal{H}}\right)$ be the Kullback-Leiber (KL) distance between the target and $p_{\mathcal{H}}$. Since $\log (a) \leq a-1 \forall a \in \mathbb{R}$, the difference between the two distances is bounded above by

$$
d\left(p^{\star}, p_{\mathcal{H}}\right)-d\left(p^{\star}, p_{\mathcal{G}}\right)=\int_{x} p^{\star}(x) \log \frac{p_{\mathcal{G}}(x)}{p_{\mathcal{H}}(x)} d x \leq \int_{x} p^{\star}(x) \frac{p_{\mathcal{G}}(x)}{p_{\mathcal{H}}(x)} d x-1 .
$$

Since the networks are identical with respect to all but nodes $i$ and $j, p_{\mathcal{G}}(x) / p_{\mathcal{H}}(x)=$ $f\left(x_{j}\right) / f\left(x_{j} \mid x_{i}\right)$, thus

$$
\int_{x} p^{\star}(x) \frac{p_{\mathcal{G}}(x)}{p_{\mathcal{H}}(x)} d x=\int_{x_{i}} \int_{x_{j}} f\left(x_{j} \mid x_{i}\right) f\left(x_{i}\right) \frac{f\left(x_{j}\right)}{f\left(x_{j} \mid x_{i}\right)} d x_{i} d x_{j}=1 .
$$

To obtain the strict inequality $\log \left(p_{\mathcal{G}}(x) / p_{\mathcal{H}}(x)\right)<p_{\mathcal{G}}(x) / p_{\mathcal{H}}(x)-1$, use that $\log (a)<$ $a-1 \Leftrightarrow a \neq 1$ and the factorization of the joint density according to both $\mathcal{G}$ and $\mathcal{H}$. Then $f\left(x_{j}\right) / f\left(x_{j} \mid x_{i}\right) \neq 1$ if and only if the densities are different for an uncountable number of points. This holds if the true regression coefficient $\beta_{j i} \neq 0$, since this implies using two normal distributions centered at different locations when $X_{i}$ is not a point mass at zero.

Next, we obtain the conditions under which the MSE decreases when adding an edge. 
Proposition 2 Suppose DAG $\mathcal{H}$ extends $\mathcal{G}$ by adding the edge $j \leftarrow i$. If for node $j,\left|\omega^{t} \delta\right|$ $>\sqrt{\omega^{t} Q^{-1} \omega}$ then $\sum_{l=1}^{p} M S E_{\mathcal{G}}\left(\hat{\mu}_{l ; S_{l}}\right)>\sum_{l=1}^{p} M S E_{\mathcal{H}}\left(\hat{\mu}_{l ; S_{l}}\right)$.

Proof The proof follows from Theorem 5.3 of Claeskens and Hjort (2008a, p. 127) since the inequality is based on a decomposable criterion, and since the two graphs are identical with respect to all other relations (that is, the nodewise MSEs will be identical for the identical relations). Thus the stated inequality will hold if for node $j, \operatorname{MSE}_{\mathcal{G}}\left(\hat{\mu}_{j ; \emptyset}\right)>\operatorname{MSE}_{\mathcal{H}}\left(\hat{\mu}_{j ;\{i\}}\right)$, and this is true if the condition in the lemma holds; otherwise if the condition does not hold then adding the arrow will not necessarily decrease the mean square error.

\section{Search Algorithms}

For the following discussion, we assume that the data are standardized to have mean 0 and variance 1 . While this is not required for the FIC algorithm at any step, it ensures a higher degree of comparability between different models.

\subsection{Algorithm for DAGs}

For the estimation of the DAG, we work with nodewise focus values. The focus of interest is the mean of a particular $X_{j}$ at covariate position $x_{p a\left(x_{j}\right)}=\left(x_{i}: i \in S_{j} \subseteq\{\mathcal{V} \backslash j\}\right)$,

$$
\mu_{j}=\mu(\sigma, \gamma ; x)=x_{p a\left(x_{j}\right)}^{T} \gamma,
$$

assuming at this stage, that all other nodes are allowed to be parents of a particular node $j$, although reducing this set on a priori knowledge reduces the necessary computing time and does not impede the algorithm in any way.

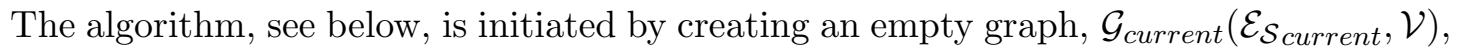
where for each node, an intercept-only model is fitted. $\mathcal{S}_{\text {current }}$ is thus at this stage $\mathcal{S}=$ $\left\{S_{1}, \ldots, S_{p} \mid S_{1}=S_{2}=\ldots=S_{p}=\emptyset\right\}$ and $\mathcal{E}_{\mathcal{S} \text { current }}=\emptyset$. For each node $j \in \mathcal{V}$, all considered models for that node are fit and the corresponding $\widehat{\mathrm{MSE}}\left(\hat{\mu}_{j ; S_{j}}\right)$ are computed. To make quantities more comparable, we standardize all the obtained $\widehat{\mathrm{MSE}}$ s per node to have mean 0 and variance 1 , and then select the global smallest such MSE. Say, this is the case for one of the models for node $j$. The empty model is updated by augmenting both the $\mathcal{E}_{\mathcal{S}_{\text {current }}}$ and $\mathcal{S}_{\text {current }}$ set to contain all the identified edges and the corresponding $S_{j}$ set. The augmented set becomes the edge set of the new updated model, and the current benchmark score is updated as well. Next, all models for node $j$ are omitted and the smallest MSE is searched over the remaining models, checking the validity of the constraints imposed by the DAG structure.

A small example might make the algorithm clearer. Consider a 3-node network based on variables $X_{1}, X_{2}$ and $X_{3}$. After fitting a list of possible models, say that for each of these variables separately the best models (intercepts have been omitted, since they are not strictly necessary, because of using centered data) are:

$$
\begin{array}{lll}
X_{1}=\beta_{12} X_{2}+\beta_{13} X_{3} & \text { with } S_{1}=\{2,3\} \text { and } \operatorname{MSE}\left(\hat{\mu}_{1 ; S_{1}}\right)=-0.5 \\
X_{2}=\beta_{23} X_{3} & \text { with } S_{2}=\{3\} \text { and } \operatorname{MSE}\left(\hat{\mu}_{2 ; S_{2}}\right)=-0.2 \\
X_{3}=\beta_{31} X_{1} & \text { with } S_{3}=\{1\} \text { and } \operatorname{MSE}\left(\hat{\mu}_{3 ; S_{3}}\right)=-0.3 .
\end{array}
$$




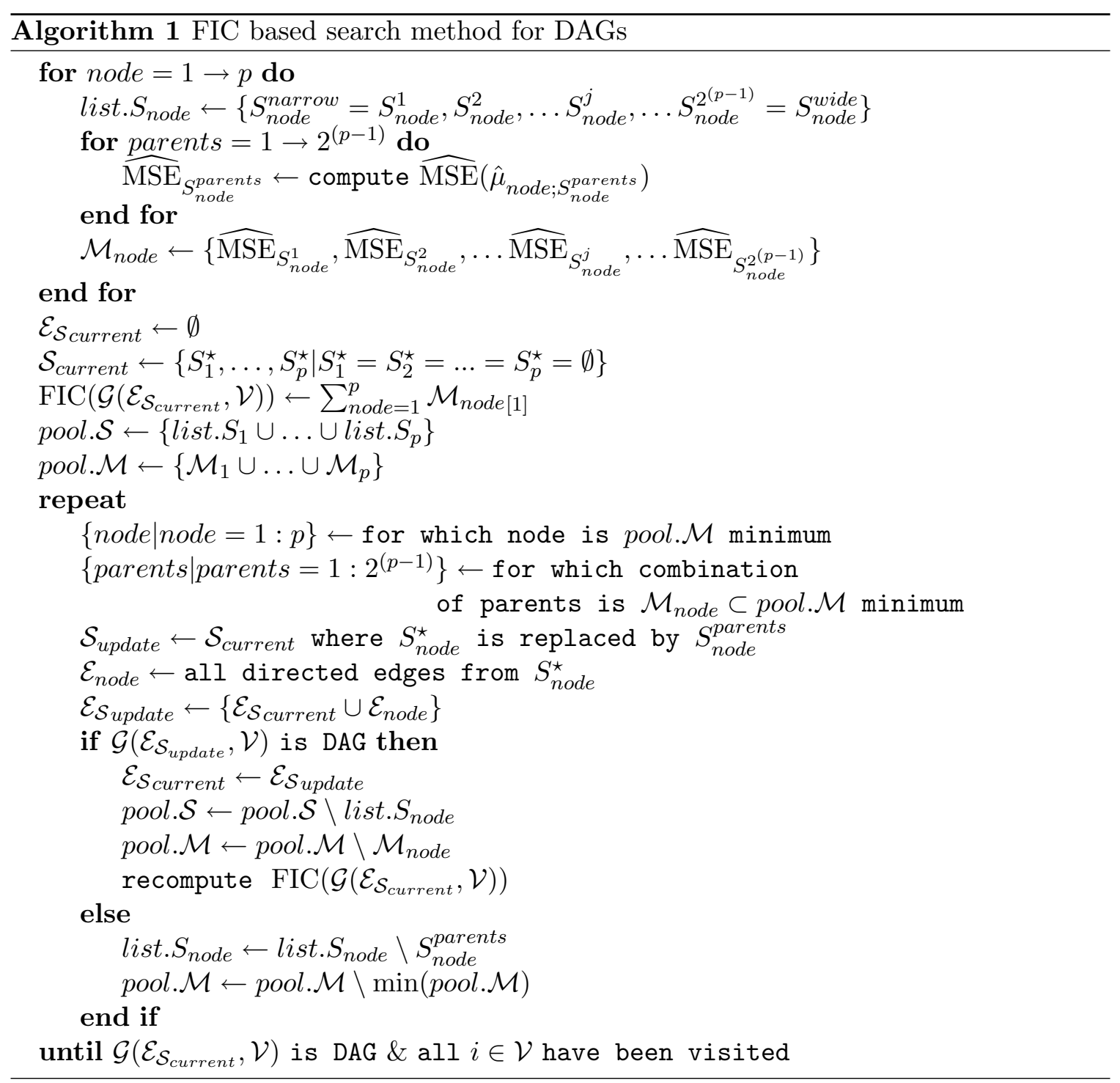

Since the model for $X_{1}$ provides the smallest standardized MSE, the update results in $\mathcal{S}_{\text {updated }}=\left\{S_{1}, S_{2}, S_{3} \mid S_{1}=\{2,3\}, S_{2}=S_{3}=\emptyset\right\}$ and $\mathcal{E}_{\mathcal{S}_{\text {updated }}}=\left\{\mathcal{E}_{\mathcal{S}_{\text {current }}} \cup(1,2) \cup(1,3)\right\}$. The updated FIC scores are based on $\mathcal{S}_{\text {updated }}$. The estimated network at this first step is $X_{2} \rightarrow X_{1} \leftarrow X_{3}$. In the next stage, we search for the best model in MSE sense for any of the remaining vertices which, when updating the working model, still results in a DAG. Once such a model is found, $\mathcal{E}_{\mathcal{S}_{\text {current }}}, \mathcal{S}_{\text {current }}$ and the benchmark score are updated accordingly. The procedure is repeated until all nodes have been visited. In the example, based on the above MSEs, the network would be updated by introducing the parents of $X_{3}$. However, this would create a feedback relation between $X_{1}$ and $X_{3}$ and since this violates the DAG assumptions, a second best model is searched for $X_{3}$. The new system of equations when inputting the second best model for $X_{3}$ becomes:

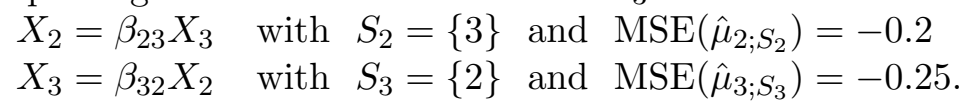


Again updating by inputting the parents of $X_{3}$ seems to be the best decision and respects the DAG constraints. The new structure adds to the previous DAG (i.e. $X_{2} \rightarrow X_{1} \leftarrow X_{3}$ ) the directed arrow $X_{2} \rightarrow X_{3}$. For the remaining $X_{2}$ node, inputing $X_{3}$ as its parent, creates a feedback loop between the two nodes, and so the decision to visit the second best model is made. Assume the second best model contains as parents only $X_{1}$. This creates again a feedback loop between $X_{1}$ and $X_{2}$ and so the third best model is visited, which, say, is an intercept model. At this stage we stop because none of the 'better-scoring' models respected the imposed DAG constraints. The final estimated model, is thus equivalent to the model from the previous step. The procedure searches at every stage of the algorithm for the best model in MSE sense that respects the DAG constraints. Since the initial search is nodewise, the verification for DAG constraints limits the possible models.

The algorithm produces at each stage a model with smaller FIC than at the previous stage, which is proved next.

Lemma 3 Given a set of nodes $\mathcal{V}$, under the local misspecification assumption, a model $S$ for each node $j \in \mathcal{V}$ can be constructed such that when updating, $\operatorname{FIC}\left(\mathcal{G}\left(\mathcal{E}_{\mathcal{S}_{\text {updated }}}, \mathcal{V}\right)\right) \leq$

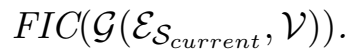

Proof The proof follows from observing that at each step the decision is made to introduce in the estimated graph, the parents of a node $j$ when it decreases the current FIC, and respects also the acyclicity condition. If the updated FIC would increase by this addition of edges, then the decision taken is to search further until a model that decreases the score or at least does not worsen it, is found. If no such model that respects acyclicity conditions can be found, then there will always exist a model, the intercept model (which amounts to node $j$ not having any parents) that will satisfy both the acyclicity condition as it will also not worsen $\operatorname{FIC}\left(\mathcal{G}\left(\mathcal{E}_{\mathcal{S}_{\text {current }}}, \mathcal{V}\right)\right)$.

There is some similarity with 'algorithm B' (Buntine, 1991) and 'algorithm K2' (Cooper and Herskovits, 1992), but important differences are present. First, the FIC based algorithm does not need any ordering of the nodes, and second, in each step more than one parent can be identified at once. It is still a greedy approach but one that pays attention to complex combinations of potential parents.

By the introduction of the focus and directing the search towards models with small mean squared error, we do not expect in the end a final model that comes close to the underlying data generating process. In some particular cases, the models identified by FIC and any other algorithm that tries to identify the hidden structure which generated the data, might come close or identical, but it is not required, and such cases might be judged as mere coincidences, rather than a stable and well behaved pattern.

We state a natural extension of the FIC algorithm for DAGs to the class of AGs that accommodates latent confounders. More specifically, we proceed in three major steps:

(i) obtain a DAG structure which specifies the set of parents for each node;

(ii) detect pairs of nodes which do not have any edge between them, and select those for which adding a $\leftrightarrow$ edge results in an AG; 
(iii) place bidirected edges between one or more of the selected pairs of nodes, say $i \leftrightarrow j$, and recompute $\operatorname{FIC}\left(\mathcal{G}\left(\mathcal{E}_{\mathcal{S}}, \mathcal{V}\right)\right)$ allowing for correlation between residuals $\epsilon_{i}$ and $\epsilon_{j}$, see Section 2.3;

If the recomputed score is better than the score for the DAG structure that was the starting point, then the AG model that allows for the existence of latent confounders will be preferred. For large networks, there might exist a large number of couples $i$ and $j$ were bidirected edges could be set and one might search for all such combinations of edges, or one might predetermine beforehand a specified number of latent variables which are allowed to enter the model. This seems to be a beneficial idea, as often in practice one expects some confounding variables to be present and unmeasured, but not too many, otherwise a wider research design setting would be beneficial.

\subsection{Algorithm for GGMs}

For the estimation of an undirected graphical structure, the same procedure of sequentially fitting a series of node-wise regressions is performed, and for each node, the set of possible predictors is constructed to contain all other possible predictors (if no exclusion can be $a$ priori performed).

Once every node has a list of plausible predictors that would minimize the MSE of the studied focus, the 'AND' rule is applied based on (1). That is, an undirected arc is set between two nodes if each of the nodes is each other's parent in the selected model having the smallest standardized FIC values, see Algorithm 2.

In the same hypothetical three-node example as in Section 4.1, we now search for a GGM. For this particular application, starting from the best nodewise models, only one relation respects the 'AND' rule, and that is $X_{3} \rightarrow X_{1}$ and $X_{3} \leftarrow X_{1}$. The updating, from an empty graph, consists in inputting an undirected arrow only between nodes 1 and 3 , as there are no other relations (between nodes 2 and 3 or between 2 and 1 ) that satisfy the GGM constraints.

\section{Numerical Results: Simulated Datasets}

After a short description of the competitive methods and settings that were created for comparison, we present the results obtained on simulated data in the DAG and GGM case. Afterwards, all methods are applied on benchmark datasets.

\subsection{Competitive Methods}

For comparison purposes, some competing algorithms have been tested. In the case of DAGs, the hill climbing (HC) algorithm (in conjunction with AIC and BIC) (see Russell and Norvig, 1995) and the PC algorithm (Spirtes et al., 2000) using partial correlation tests for independence testing, with an additional orientation procedure as in Dor and Tarsi (1992), have been applied. For the GGM setting we have compared with SIN (Drton and Perlman, 2004) and graphical lasso (Friedman et al., 2008).

The PC algorithm starts from a fully connected undirected graph and tests the existence of a directed arrow by testing for independence. Next, orientations are found according to specific rules. This results in a partial DAG (PDAG) as for some of the undirected edges 


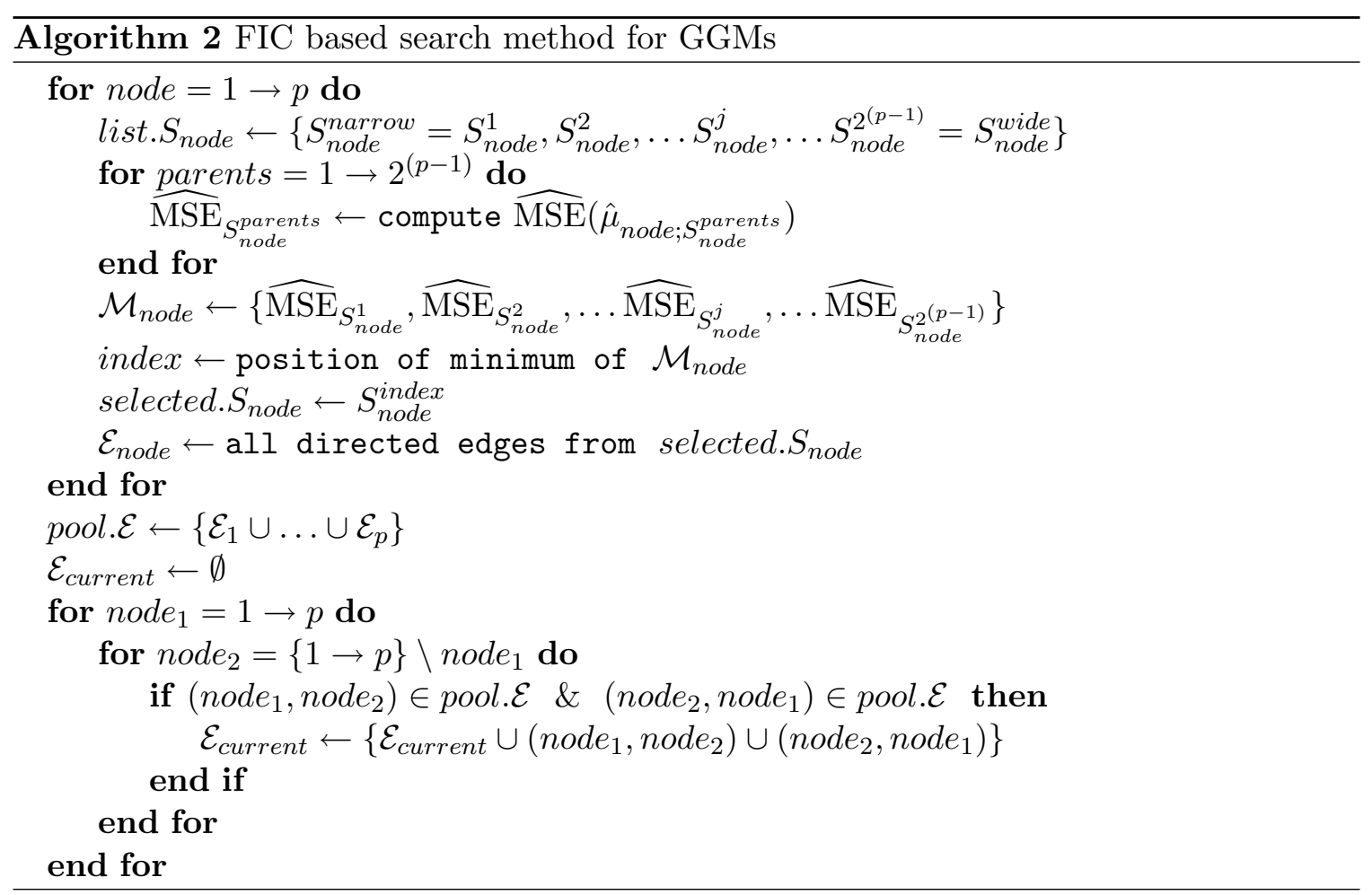

the directionality cannot be uniquely determined. The algorithm of Dor and Tarsi (1992) takes the PDAG as input and returns a DAG if the partial DAG admits such an extension, otherwise the algorithm returns the PDAG.

Hill-climbing starts from an empty graph and adds, in a first step, the best directed single edge in the graph, according to the corresponding AIC or BIC values. If the graph contains at least one edge, a decision among three possible modifications is made: either add an extra arrow between a pair of the remaining nodes, delete one of the present edges or reverse it. The same process continues until the AIC or BIC scores cannot be improved anymore. The algorithm can be seen as a 'greedy' search strategy.

Drton and Perlman (2004) introduced the SIN methodology for GGMs, that was later extended to DAGs and chain graphs in Drton and Perlman (2008), which is based on simultaneous testing whether all partial correlations $\rho_{i j}$ between pairs of variables $\left(X_{i}, X_{j}\right)$ conditioned on all remaining variables $X_{\mathcal{V} \backslash\{i, j\}}$ are zero. The size of the obtained $p$-values determines a separation in three groups: $S$ ignificant (edges that should be included in the estimated $\mathcal{G}$ ), Indeterminate (edges for which inclusion might be a decision if a less conservative level were to be chosen) and $N$ on-significant (edges that should not be included).

In the context of high-dimensional data $(n<p)$, Meinshausen and Bühlmann (2006) introduced a procedure based on a series of nodewise lasso regressions to obtain an estimate of a GGM structure. Later, Friedman et al. (2008) and Witten et al. (2011) extended the lasso procedure to multivariate density estimation, by using ideas of penalization directly on the $\Sigma^{-1}$ matrix, and labeled the resulting procedure as graphical lasso (GLasso), concluding as in Yuan and Lin (2007) that the nodewise procedure is just an approximation to the GLasso solution. GLasso maximizes the penalized log-likelihood of the data, which is up to 
a constant,

$$
\log \operatorname{det} \Sigma^{-1}-\operatorname{trace}\left(W \Sigma^{-1}\right)-\lambda\left\|\Sigma^{-1}\right\|_{1}
$$

over positive definite matrices $\Sigma^{-1}$, where $W$ is the sample covariance matrix and $\left\|\Sigma^{-1}\right\|_{1}$ is the $l_{1}$ norm i.e., the sum of absolute values of the entries in the $\Sigma^{-1}$ matrix. Varying $\lambda$, the amount of penalization on the $l_{1}$ norm, causes some elements of the matrix to be set to 0 . Yuan and Lin (2007) proposed the application of the BIC criterion in selecting the amount of regularization, which is also what we use.

\subsection{Simulation Study Setup}

Different scenarios have been constructed using sample sizes equal to 50, 100 and 200 and networks having either 5 or 8 nodes. The 5-node network contains either 5 (low) or 10 (high) edges, while the 8-node network contains either 10 (low) or 20 (high) edges. All edges are chosen arbitrarily, and are kept fixed, such that the topology of the networks remains the same throughout each simulation run. For each parent, the corresponding weight (regression coefficient) is randomly sampled from a uniform distribution with range $[-2,2]$, and noise is generated from a normal distribution $N\left(0, \sigma^{2}\right)$ with $\sigma=1,2,3$ or 5 . A graphical illustration of the four networks can be seen in Figure 1.

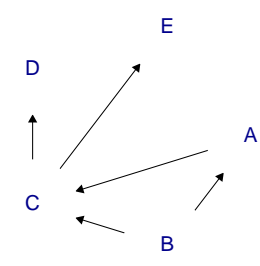

(a)

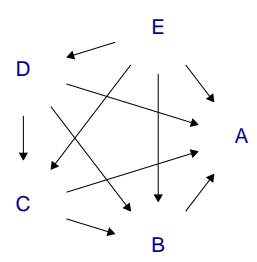

(b)

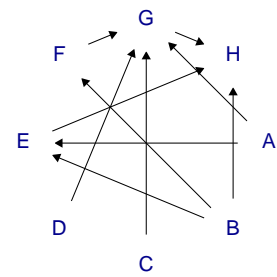

(c)

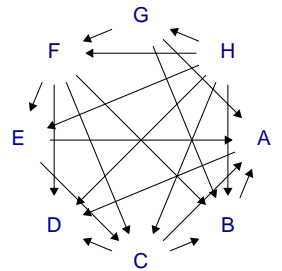

(d)

Figure 1: True DAG structure on which simulations are based.

As an example, the simulation setting with 5 nodes and 5 edges that has been used, see Figure 1(a), is represented as follows:

$$
A=\beta_{1} B+\epsilon_{A} ; C=\beta_{2} A+\beta_{3} B+\epsilon_{C} ; D=\beta_{4} C+\epsilon_{D} ; E=\beta_{5} C+\epsilon_{E} .
$$

Both a DAG and a GGM have been selected on each simulated dataset using the algorithms in Section 4 for the focused search. The number of simulation runs has been set to 1000, and at each simulation run, for DAGs a leave-one-out cross-validation (LOO CV) using the average squared prediction error as cost function, has been performed at each node. The overall final prediction error for the network $\mathcal{G}(\mathcal{E}, \mathcal{V})$ that has been estimated, is constructed by adding all such nodewise average squared prediction errors,

$$
\operatorname{PE}(\mathcal{G}(\mathcal{E}, \mathcal{V}))=n^{-1} \sum_{i=1}^{p} \sum_{k=1}^{n}\left(X_{i}^{k}-\widehat{X_{i}^{-k}}\right)^{2}
$$

where $X_{i}^{k}$ represents the observed value of the $k$-th sample case for node $i$ and $\widehat{X_{i}^{-k}}$ represents the fitted value for the same sample case $k$, on the same node $i$, but where the model 
has been fitted using all other sample cases, except case $k$. For the GGM 5-fold CV has been used, where the dataset is divided into five parts, a training is performed on $\left(1-\frac{1}{5}\right)$ parts of the sample and then prediction errors are evaluated on the remaining part of the sample, similar to the above LOO technique.

Alongside prediction errors, the true positive rate $(\mathrm{TPR}=$ number of correctly found edges by the estimated graph, divided by the total number of true edges), false positive rate $(\mathrm{FPR}=$ number of incorrectly found edges divided by the total number of true non-existent edges) and true discovery rate $(\mathrm{TDR}=$ number of correctly found edges divided by the total number of estimated edges) are also investigated.

\subsection{Simulation Study Results}

For the 5-node network for DAGs, see Table 1, the differences in predictive power are not large especially between FIC and HC performance, but on the larger network, larger differences occur. For the smaller network the PE decreases as the number of edges rises while in the larger network, increasing the number of edges deteriorates the overall prediction errors. This happens because increasing the number of edges in the small network, results in a graph whose skeleton is completely connected, and thus the setting is easier for the algorithms. However, at all levels, FIC produces comparable or better PE than the competing algorithms. For a given noise level, increasing the sample size decreases the prediction errors for all methods, with the PC algorithm having the largest such decrease.

Table 2 indicates that in all settings, with respect to TPR, the performance of the FIC algorithm is better (in some cases by quite a large margin) or comparable to that of the competitors (see also Figure 3). It is thus apparent that the FIC algorithm is on average closer to correctly identifying which two nodes should be truly connected, however it might reverse the true directionality if this results in better predictions. In all settings, FIC seems to prefer larger models with more false positives, as this helps reducing the estimated MSE, and almost always it produces lower TDR.

The conclusion is that for DAGs, the FIC algorithm on average produces smaller prediction errors, seems to better identify which two nodes should be connected, but more often reverses the true directionality to serve its purpose.

In the top part of Table 1, an estimated average FIC model has been used where the FIC values are averaged over all observations. The FIC method easily allows to select individual specific models, where in the specification of the focus only the covariate information of that individual is used. We have compared prediction errors obtained from an estimated model for a particular subject with the prediction errors obtained from the $\mathrm{HC}$ and $\mathrm{PC}$ estimated models, see Figure 2. For these simulations, we have obtained, on average, prediction errors that were roughly $10 \%$ lower when compared to the best competitor. In some settings the obtained prediction errors were up to $20-23 \%$ lower (see Table 1 middle panel).

In the GGM case, for low sample sizes with a low number of edges, GLasso performs best, followed by the FIC algorithm, but increasing the number of edges places FIC as the best algorithm, with GLasso deteriorating by a large margin. On the same small network with larger sample sizes, the FIC selected graph performs either better or similar to all the other estimated graphs, while for the larger 8-node network, in almost all settings FIC performs better. In most of the simulated settings, TPR was best for GLasso, followed second by 


\begin{tabular}{|c|c|c|c|c|c|c|c|c|c|c|c|c|}
\hline \multirow[b]{3}{*}{$n$} & \multirow[b]{3}{*}{$\sigma$} & \multirow[b]{3}{*}{$\mathrm{E}$} & \multicolumn{5}{|c|}{ 5-node network } & \multicolumn{5}{|c|}{ 8-node network } \\
\hline & & & \multirow[b]{2}{*}{ FIC } & \multicolumn{2}{|c|}{$\mathrm{HC}$} & \multicolumn{2}{|c|}{$\mathrm{PC}(\alpha=)$} & \multirow[b]{2}{*}{ FIC } & \multicolumn{2}{|c|}{$\mathrm{HC}$} & \multicolumn{2}{|c|}{$\mathrm{PC}(\alpha=)$} \\
\hline & & & & $\mathrm{BIC}$ & $\mathrm{AIC}$ & .1 & .05 & & $\mathrm{BIC}$ & AIC & .1 & .05 \\
\hline \multicolumn{13}{|c|}{ DAG: model based on average FIC scores across subjects } \\
\hline \multirow[t]{4}{*}{50} & 3 & Low & 2.64 & 2.67 & 2.66 & 2.99 & 3.09 & 4.72 & 5.24 & 5.22 & 5.71 & 5.80 \\
\hline & & High & 2.10 & 2.26 & 2.25 & 2.72 & 2.77 & 4.89 & 5.07 & 5.05 & 5.67 & 5.81 \\
\hline & 5 & Low & 2.61 & 2.63 & 2.62 & 2.96 & 3.06 & 4.72 & 5.24 & 5.21 & 5.71 & 5.80 \\
\hline & & High & 2.13 & 2.28 & 2.28 & 2.76 & 2.82 & 4.89 & 5.07 & 5.05 & 5.67 & 5.81 \\
\hline \multirow[t]{4}{*}{100} & 3 & Low & 2.58 & 2.60 & 2.60 & 2.81 & 2.84 & 4.63 & 5.19 & 5.17 & 5.55 & 5.59 \\
\hline & & High & 2.06 & 2.19 & 2.19 & 2.57 & 2.63 & 4.82 & 5.02 & 5.00 & 5.43 & 5.49 \\
\hline & 5 & Low & 2.63 & 2.65 & 2.64 & 2.86 & 2.89 & 4.69 & 5.22 & 5.21 & 5.56 & 5.59 \\
\hline & & High & 2.08 & 2.22 & 2.22 & 2.61 & 2.66 & 4.82 & 5.01 & 5.00 & 5.43 & 5.48 \\
\hline \multirow[t]{4}{*}{200} & 3 & Low & 2.52 & 2.55 & 2.55 & 2.69 & 2.71 & 4.59 & 5.18 & 5.17 & 5.47 & 5.49 \\
\hline & & High & 2.04 & 2.17 & 2.17 & 2.49 & 2.54 & 4.79 & 4.97 & 4.96 & 5.30 & 5.33 \\
\hline & 5 & Low & 2.50 & 2.51 & 2.51 & 2.67 & 2.68 & 4.56 & 5.14 & 5.13 & 5.43 & 5.45 \\
\hline & & High & 2.03 & 2.17 & 2.17 & 2.51 & 2.54 & 4.79 & 4.97 & 4.96 & 5.29 & 5.33 \\
\hline \multicolumn{13}{|c|}{ DAG: model based on subject specific FIC scores } \\
\hline \multirow[t]{4}{*}{50} & 3 & Low & 2.37 & 2.55 & 2.51 & 2.81 & 2.91 & 3.63 & 4.95 & 4.87 & 5.36 & 5.50 \\
\hline & & High & 1.88 & 2.14 & 2.11 & 2.60 & 2.68 & 4.08 & 4.78 & 4.70 & 5.33 & 5.50 \\
\hline & 5 & Low & 2.40 & 2.56 & 2.53 & 2.83 & 2.93 & 3.61 & 4.93 & 4.86 & 5.33 & 5.45 \\
\hline & & High & 1.91 & 2.14 & 2.12 & 2.63 & 2.70 & 4.04 & 4.71 & 4.64 & 5.27 & 5.44 \\
\hline \multirow[t]{4}{*}{100} & 3 & Low & 2.52 & 2.50 & 2.50 & 2.67 & 2.73 & 3.81 & 4.98 & 4.95 & 5.40 & 5.40 \\
\hline & & High & 1.93 & 2.12 & 2.12 & 2.55 & 2.59 & 4.44 & 4.87 & 4.82 & 5.28 & 5.33 \\
\hline & 5 & Low & 2.52 & 2.48 & 2.48 & 2.67 & 2.73 & 3.80 & 4.97 & 4.94 & 5.38 & 5.38 \\
\hline & & High & 1.92 & 2.11 & 2.10 & 2.52 & 2.56 & 4.44 & 4.85 & 4.82 & 5.27 & 5.33 \\
\hline \multirow[t]{4}{*}{200} & 3 & Low & 2.51 & 2.43 & 2.43 & 2.59 & 2.61 & 4.05 & 5.16 & 5.15 & 5.41 & 5.44 \\
\hline & & High & 1.90 & 2.05 & 2.04 & 2.37 & 2.44 & 4.67 & 5.10 & 5.08 & 5.40 & 5.46 \\
\hline & 5 & Low & 2.48 & 2.40 & 2.40 & 2.56 & 2.58 & 4.04 & 5.17 & 5.16 & 5.41 & 5.44 \\
\hline & & High & 1.88 & 2.03 & 2.02 & 2.34 & 2.42 & 4.63 & 5.06 & 5.04 & 5.36 & 5.43 \\
\hline \multirow[t]{2}{*}{ GGM } & & & & \multicolumn{3}{|c|}{$\operatorname{SIN}(\alpha=)$} & GL & & & $\mathrm{N}(\alpha=$ & & GL \\
\hline & & & FIC & .25 & .15 & .05 & $\mathrm{BIC}$ & FIC & .25 & .15 & .05 & $\mathrm{BIC}$ \\
\hline 50 & 3 & Low & 1.87 & 2.06 & 2.15 & 2.30 & 1.80 & 3.25 & 3.73 & 3.88 & 4.16 & 3.77 \\
\hline & & High & 1.08 & 1.16 & 1.23 & 1.40 & 1.32 & 3.84 & 4.30 & 4.42 & 4.68 & 3.83 \\
\hline & 5 & Low & 1.83 & 2.01 & 2.09 & 2.23 & 1.75 & 3.23 & 3.74 & 3.89 & 4.17 & 3.76 \\
\hline & & High & 1.07 & 1.15 & 1.22 & 1.39 & 1.32 & 3.82 & 4.28 & 4.39 & 4.64 & 3.82 \\
\hline 100 & 3 & Low & 1.67 & 1.76 & 1.80 & 1.90 & 1.74 & 2.77 & 2.95 & 3.02 & 3.18 & 3.67 \\
\hline & & High & 0.87 & 0.89 & 0.91 & 0.97 & 1.24 & 3.50 & 3.73 & 3.80 & 3.94 & 3.78 \\
\hline & 5 & Low & 1.63 & 1.71 & 1.76 & 1.85 & 1.69 & 2.72 & 2.90 & 2.98 & 3.13 & 3.66 \\
\hline & & High & 0.84 & 0.86 & 0.88 & 0.95 & 1.22 & 3.52 & 3.76 & 3.82 & 3.96 & 3.79 \\
\hline 200 & 3 & Low & 1.51 & 1.53 & 1.55 & 1.59 & 1.63 & 2.59 & 2.64 & 2.66 & 2.72 & 3.65 \\
\hline & & High & 0.74 & 0.75 & 0.75 & 0.77 & 1.17 & 3.33 & 3.42 & 3.45 & 3.52 & 3.72 \\
\hline & 5 & Low & 1.56 & 1.59 & 1.60 & 1.64 & 1.69 & 2.55 & 2.61 & 2.63 & 2.69 & 3.64 \\
\hline & & High & 0.74 & 0.75 & 0.75 & 0.77 & 1.16 & 3.38 & 3.48 & 3.52 & 3.59 & 3.77 \\
\hline
\end{tabular}

Table 1: Average LOO CV squared prediction errors (for DAGs based on average FIC), average squared prediction errors (for subject specific DAGs) and average 5-fold CV squared prediction errors (for GGMs). Networks containing 5, 10 (low,high) edges (E) for the 5-node network, or 10, 20 (low,high) edges for the 8-node network with sample size $n$, and standard deviation $\sigma$. GL stands for GLasso. Results have been averaged over 1000 simulation runs. 


\begin{tabular}{|c|c|c|c|c|c|c|c|c|c|c|c|c|c|}
\hline \multirow[b]{3}{*}{$\mathrm{N}$} & \multirow[b]{3}{*}{$n$} & \multirow[b]{3}{*}{$\sigma$} & \multirow[b]{3}{*}{$\mathrm{E}$} & \multicolumn{5}{|c|}{ DAG skeleton } & \multicolumn{5}{|c|}{ GGM } \\
\hline & & & & \multirow[b]{2}{*}{ FIC } & \multicolumn{2}{|c|}{$\mathrm{HC}$} & \multicolumn{2}{|c|}{$\mathrm{PC}(\alpha=)$} & \multicolumn{5}{|c|}{$\operatorname{SIN}(\alpha=)$} \\
\hline & & & & & $\mathrm{BIC}$ & AIC & .1 & .05 & FIC & .25 & .15 & .05 & GLas \\
\hline \multirow[t]{18}{*}{5} & \multirow[t]{6}{*}{50} & \multirow[t]{2}{*}{2} & 5 & 0.84 & 0.81 & 0.77 & 0.63 & 0.59 & 0.74 & 0.62 & 0.57 & 0.50 & 0.90 \\
\hline & & & 10 & 0.85 & 0.75 & 0.68 & 0.47 & 0.43 & 0.74 & 0.69 & 0.64 & 0.56 & 0.82 \\
\hline & & \multirow[t]{2}{*}{3} & 5 & 0.86 & 0.82 & 0.77 & 0.63 & 0.59 & 0.74 & 0.63 & 0.58 & 0.50 & 0.90 \\
\hline & & & 10 & 0.85 & 0.75 & 0.68 & 0.47 & 0.43 & 0.74 & 0.69 & 0.64 & 0.56 & 0.82 \\
\hline & & \multirow[t]{2}{*}{5} & 5 & 0.85 & 0.82 & 0.78 & 0.63 & 0.59 & 0.74 & 0.62 & 0.57 & 0.50 & 0.90 \\
\hline & & & 10 & 0.85 & 0.76 & 0.69 & 0.47 & 0.43 & 0.74 & 0.69 & 0.64 & 0.56 & 0.82 \\
\hline & \multirow[t]{6}{*}{100} & \multirow[t]{2}{*}{2} & 5 & 0.90 & 0.87 & 0.83 & 0.73 & 0.69 & 0.83 & 0.76 & 0.73 & 0.67 & 0.91 \\
\hline & & & 10 & 0.89 & 0.82 & 0.74 & 0.54 & 0.50 & 0.81 & 0.80 & 0.76 & 0.70 & 0.82 \\
\hline & & \multirow[t]{2}{*}{3} & 5 & 0.90 & 0.87 & 0.83 & 0.72 & 0.69 & 0.83 & 0.75 & 0.72 & 0.66 & 0.92 \\
\hline & & & 10 & 0.89 & 0.81 & 0.74 & 0.55 & 0.51 & 0.81 & 0.79 & 0.75 & 0.69 & 0.82 \\
\hline & & \multirow[t]{2}{*}{5} & 5 & 0.90 & 0.87 & 0.83 & 0.72 & 0.69 & 0.83 & 0.75 & 0.72 & 0.67 & 0.91 \\
\hline & & & 10 & 0.90 & 0.82 & 0.75 & 0.54 & 0.50 & 0.82 & 0.80 & 0.76 & 0.70 & 0.82 \\
\hline & \multirow[t]{6}{*}{200} & \multirow[t]{2}{*}{2} & 5 & 0.93 & 0.92 & 0.88 & 0.80 & 0.78 & 0.89 & 0.85 & 0.83 & 0.79 & 0.91 \\
\hline & & & 10 & 0.92 & 0.86 & 0.78 & 0.61 & 0.57 & 0.87 & 0.87 & 0.84 & 0.79 & 0.82 \\
\hline & & \multirow[t]{2}{*}{3} & 5 & 0.93 & 0.91 & 0.88 & 0.80 & 0.77 & 0.88 & 0.84 & 0.82 & 0.78 & 0.92 \\
\hline & & & 10 & 0.92 & 0.86 & 0.79 & 0.61 & 0.58 & 0.87 & 0.86 & 0.84 & 0.79 & 0.82 \\
\hline & & \multirow[t]{2}{*}{5} & 5 & 0.93 & 0.91 & 0.88 & 0.80 & 0.78 & 0.88 & 0.84 & 0.82 & 0.78 & 0.91 \\
\hline & & & 10 & 0.93 & 0.86 & 0.79 & 0.61 & 0.57 & 0.87 & 0.86 & 0.84 & 0.79 & 0.83 \\
\hline 8 & 50 & 2 & 10 & 0.86 & 0.86 & 0.81 & 0.53 & 0.49 & 0.74 & 0.57 & 0.53 & 0.46 & 0.85 \\
\hline & & & 20 & 0.54 & 0.46 & 0.38 & 0.27 & 0.24 & 0.40 & 0.24 & 0.22 & 0.18 & 0.68 \\
\hline & & 3 & 10 & 0.86 & 0.87 & 0.82 & 0.53 & 0.49 & 0.73 & 0.57 & 0.53 & 0.46 & 0.84 \\
\hline & & & 20 & 0.54 & 0.46 & 0.38 & 0.27 & 0.24 & 0.39 & 0.24 & 0.22 & 0.19 & 0.68 \\
\hline & & 5 & 10 & 0.86 & 0.87 & 0.82 & 0.53 & 0.49 & 0.73 & 0.57 & 0.53 & 0.46 & 0.85 \\
\hline & & & 20 & 0.54 & 0.46 & 0.38 & 0.27 & 0.25 & 0.39 & 0.24 & 0.22 & 0.19 & 0.68 \\
\hline & 100 & 2 & 10 & 0.90 & 0.91 & 0.86 & 0.61 & 0.58 & 0.83 & 0.73 & 0.71 & 0.65 & 0.85 \\
\hline & & & 20 & 0.56 & 0.48 & 0.40 & 0.32 & 0.29 & 0.44 & 0.32 & 0.30 & 0.27 & 0.68 \\
\hline & & 3 & 10 & 0.91 & 0.90 & 0.86 & 0.62 & 0.58 & 0.83 & 0.72 & 0.70 & 0.65 & 0.85 \\
\hline & & & 20 & 0.56 & 0.47 & 0.39 & 0.32 & 0.29 & 0.44 & 0.32 & 0.30 & 0.27 & 0.68 \\
\hline & & 5 & 10 & 0.90 & 0.90 & 0.86 & 0.62 & 0.59 & 0.83 & 0.73 & 0.70 & 0.65 & 0.85 \\
\hline & & & 20 & 0.56 & 0.48 & 0.39 & 0.32 & 0.29 & 0.43 & 0.31 & 0.29 & 0.27 & 0.68 \\
\hline & 200 & 2 & 10 & 0.93 & 0.93 & 0.90 & 0.67 & 0.65 & 0.88 & 0.82 & 0.81 & 0.77 & 0.86 \\
\hline & & & 20 & 0.58 & 0.49 & 0.41 & 0.35 & 0.33 & 0.47 & 0.37 & 0.36 & 0.34 & 0.68 \\
\hline & & 3 & 10 & 0.93 & 0.93 & 0.89 & 0.67 & 0.64 & 0.88 & 0.83 & 0.81 & 0.78 & 0.86 \\
\hline & & & 20 & 0.59 & 0.49 & 0.41 & 0.35 & 0.33 & 0.47 & 0.37 & 0.36 & 0.34 & 0.68 \\
\hline & & 5 & 10 & 0.93 & 0.94 & 0.90 & 0.68 & 0.66 & 0.89 & 0.83 & 0.81 & 0.78 & 0.85 \\
\hline & & & 20 & 0.59 & 0.49 & 0.41 & 0.35 & 0.33 & 0.47 & 0.37 & 0.35 & 0.33 & 0.68 \\
\hline
\end{tabular}

Table 2: True positive rate summary using the estimated undirected skeleton of the DAG and the GGM solution. Results have been averaged over 1000 simulation runs. 


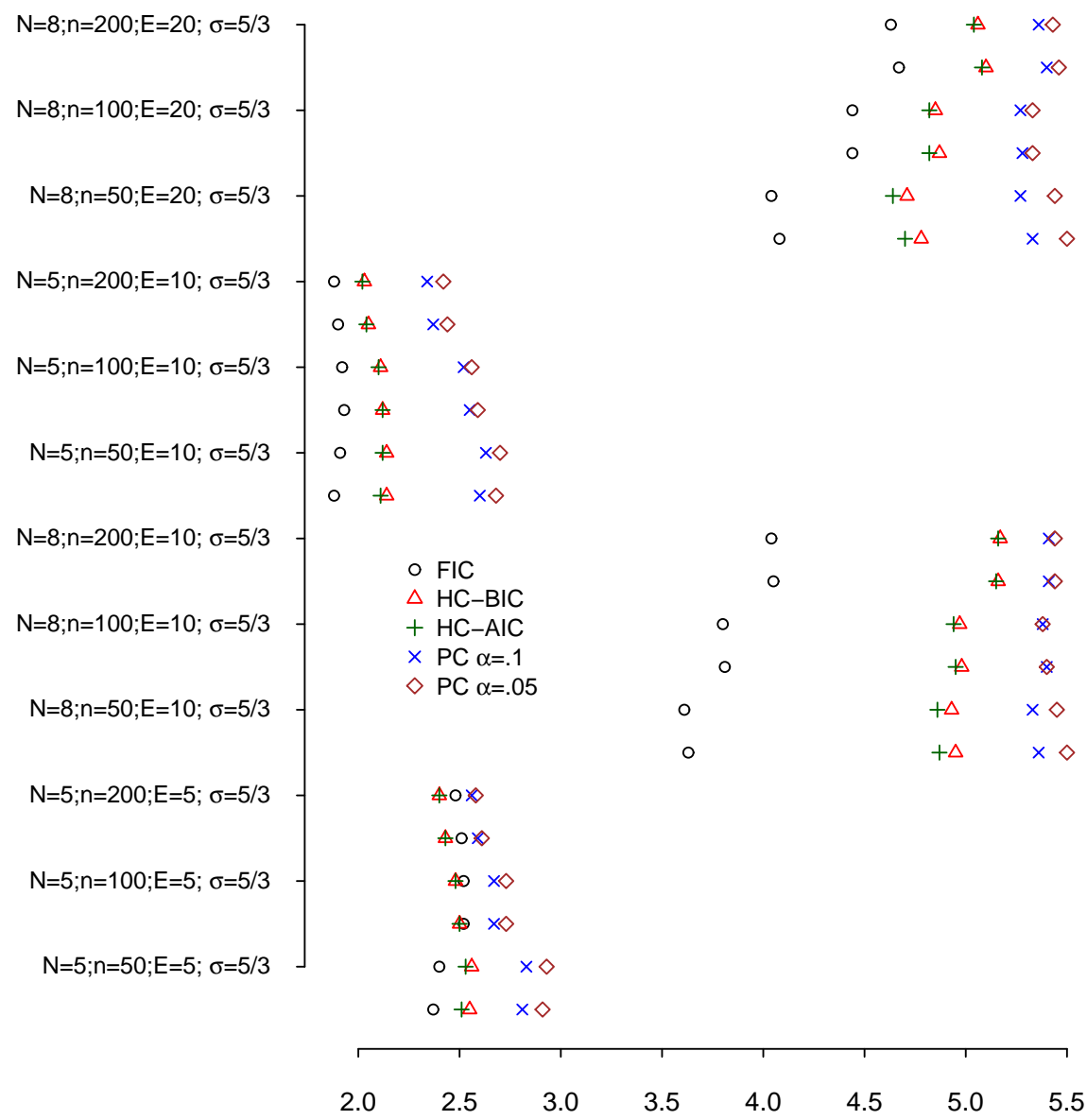

Figure 2: Average squared prediction errors for 1000 simulation runs, subject-specific DAG for different number of nodes $(\mathrm{N})$, sample sizes (n), edges $(\mathrm{E})$ and $\sigma$.

FIC. GLasso selected more false positives, followed by the FIC, while in all settings, SIN performed best in terms of TDR.

\section{Data Examples}

Five datasets are used to illustrate the method's performance. These are 'Mathematics grades' (Mardia et al., 1979); 'Glucose control' (Cox and Wermuth, 1996); 'Bone mineral content' (Edwards, 2000); 'Cork borings' (Whittaker, 1990) and 'Fret's heads' dataset (Whittaker, 1990).

\subsection{DAG Estimation for 'Mathematics Grades'.}

The 'Mathematics grades' dataset contains 88 observations on 5 variables: mechanics $(\mathrm{MECH})$, vectors (VECT), algebra (ALG), analysis (ANL) and statistics (STAT) . The objective is to discover a plausible structure between these five variables. 


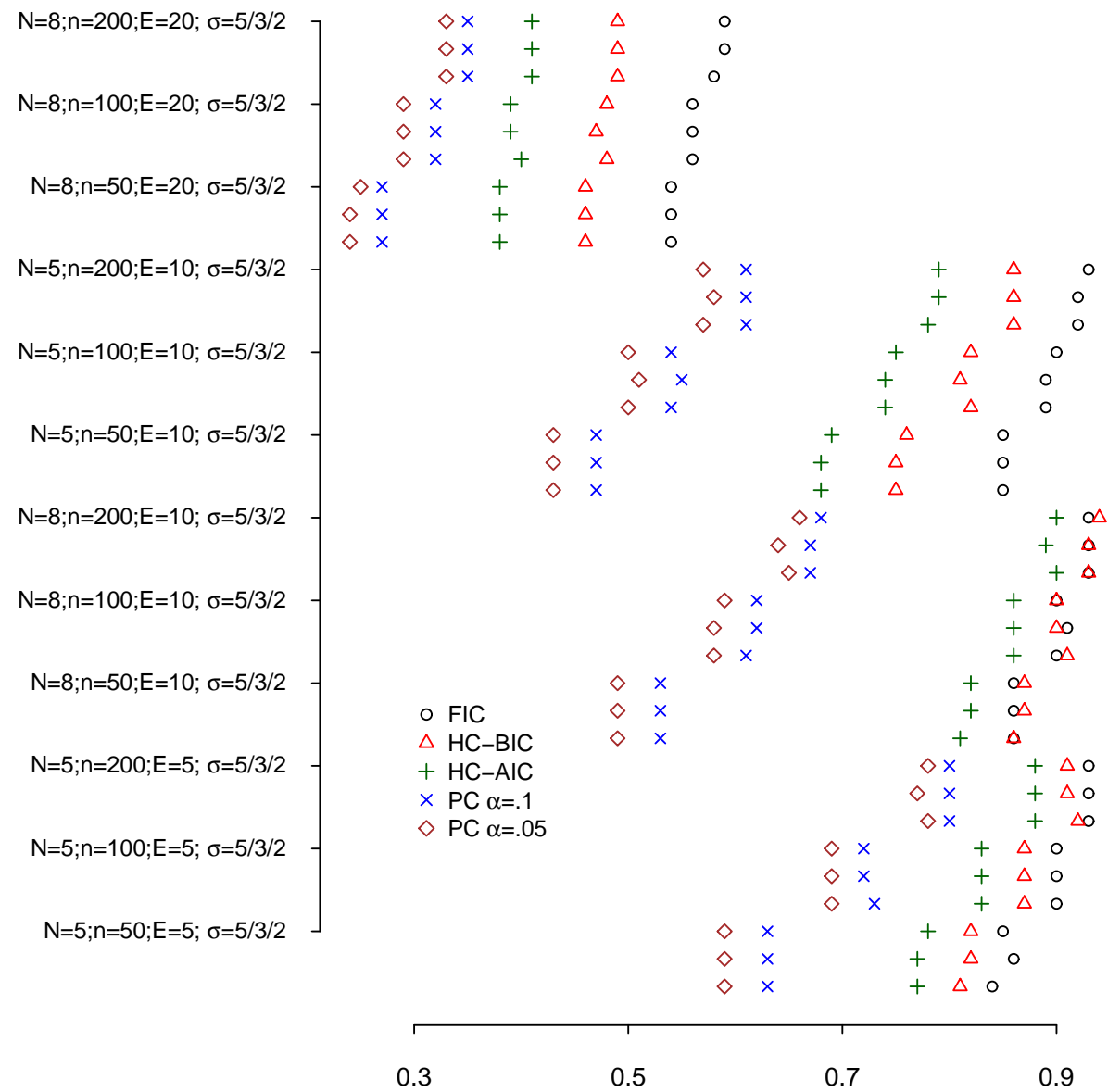

Figure 3: True positive rate summary using the estimated undirected skeleton of the DAG for different number of nodes (N), sample sizes (n), edges (E) and $\sigma$.

Figure 4 presents the selected models according to the FIC algorithms and the graphs obtained using the $\mathrm{HC}$ and $\mathrm{PC}$ method (with additional orientation for edges with undetermined directionality). Hill-climbing, as well as some variations, namely TABU and MMHC (Tsamardinos et al., 2006), all agree and identify the structure presented in Figure 4(b) as the more probable one.

The FIC selected graph was obtained by averaging the subject specific FIC values (via the subject specific covariate in the focus) for all of the subjects in the study and searching through $2^{p-1}$ combinations of parents. The selected model is presented in Figure 4(a), and suggests that, using algebra as a predictor for all other variables, as well as reversing (when compared to $\mathrm{HC}$ solution) the remaining directed arrows for statistics and mechanics, leads to smaller prediction errors (see Table 3). For all three solutions, the skeletons of the estimated networks and the set of 'immoralities' (or ' $\mathrm{v}$ '-structure) are equivalent.

The networks for two particular subjects (bottom and top grade student) are presented in Figure 4(e) and (f). The ranking between students has been done based on their corresponding overall average grades. The vectors of grades on the standardized dataset, are 


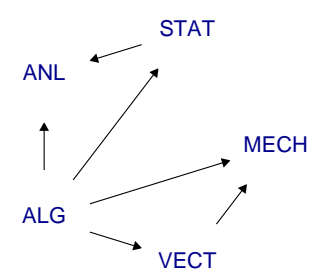

(a) FIC-DAG

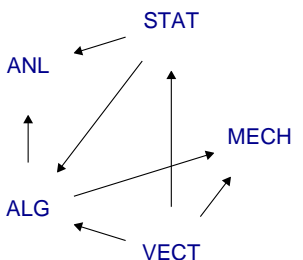

(e) FIC-DAG top student

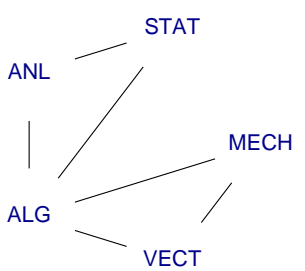

(i) FIC-GGM

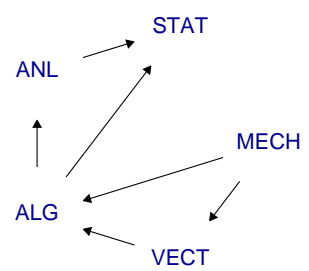

(b) $\mathrm{HC}(\mathrm{BIC})$

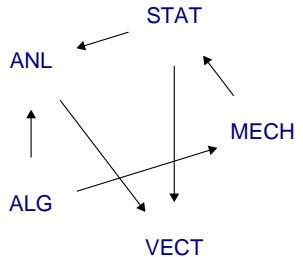

(f) FIC-DAG bottom student

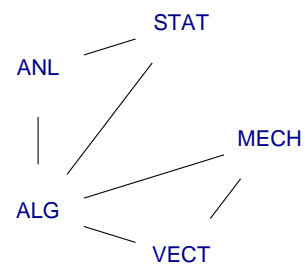

(j) $\operatorname{SIN}(\alpha=.25)$

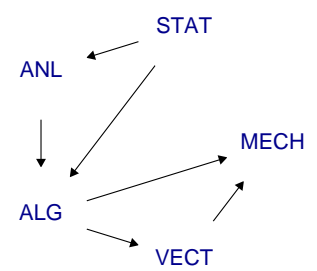

(c) $\mathrm{PC}(\alpha=.1)$

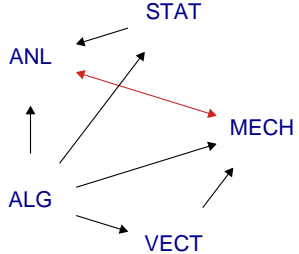

(g) FIC-AG

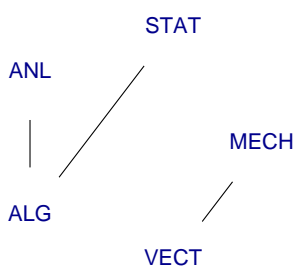

(k) $\operatorname{SIN}(\alpha=.05)$

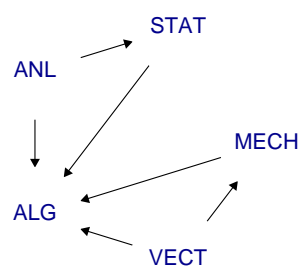

(d) FIC-DAG(with start)

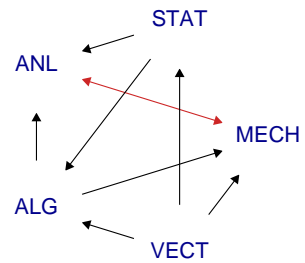

(h) FIC-AG top student

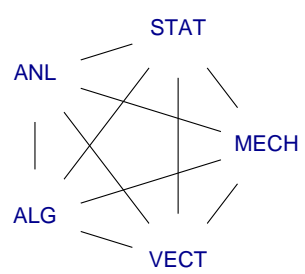

(1) GLasso(BIC)

Figure 4: 'Mathematics grades' dataset. Estimated graphs using FIC, HC and PC methods.

$(2.18,2.39,1.54,1.37,2.24)$ for the top student, and $(-1.48,0.03,-0.06,-1.46,-0.66)$ for the bottom rank student. These are used as covariate input in the focus.

To ease understanding, we present for variable ALG for the top student, how the procedure works. First we define the focus $\mu$ to be the mean of ALG at covariate position $(\mathrm{MECH}, \mathrm{VECT}, \mathrm{ANL}, \mathrm{STAT})=(2.18,2.39,1.37,2.24)$. We fit the normal linear regression model

$$
\mathrm{ALG}_{k}=\beta_{0}+\beta_{1} \mathrm{MECH}_{k}+\beta_{2} \mathrm{VECT}_{k}+\beta_{3} \mathrm{ANL}_{k}+\beta_{4} \mathrm{STAT}_{k}+\epsilon_{k}, \quad k=1, \ldots, n .
$$

The predictors are separated in a set $X$ representing all variables which should be entered based on a priori knowledge, and a set $Z$ containing all variables for which variable selection is to be performed. In this example, $X$ should contain only the intercept, because all other variables are possible predictors which are subject to model selection. 
The empirical versions of the previous expressions for $J$ and $Q$ matrices, from section 3 are given by

$$
J_{n}=n^{-1} \sum_{i=1}^{n} \frac{1}{\sigma^{2}}\left(\begin{array}{cc}
2 & 0 \\
0 & \Sigma_{n}
\end{array}\right) ; Q_{n}=\sigma^{2}\left(n^{-1} Z^{T}\left(I-X\left(X^{T} X\right)^{-1} X^{T}\right) Z\right)^{-1},
$$

where $\Sigma_{n}=\left(\begin{array}{cc}\Sigma_{n, 00} & \Sigma_{n, 01} \\ \Sigma_{n, 10} & \Sigma_{n, 11}\end{array}\right)=n^{-1}\left(\begin{array}{cc}X^{T} X & X^{T} Z \\ Z^{T} X & Z^{T} Z\end{array}\right)$. A subsequent derivation reveals

$$
\omega=Z^{T} X\left(X^{T} X\right)^{-1} x-z,
$$

where $x$ and $z$ are the prespecified covariate values corresponding to $X$ and $Z$, in the example above, the grades for the top student on the remaining four variables. Since the FIC score depends on the $\omega$ vector, which is modified each time a different value is specified for $x$ or $z$, different FIC scores may be obtained for different covariate positions, and so for each sample case, a vector of estimated FIC values can be constructed for each combination of possible parents.

When comparing the estimated networks for top and bottom rank student, it is apparent that relations such as $A L G \rightarrow A N L \leftarrow S T A T$, or $A L G \rightarrow M E C H$ are common, while the remaining ones are quite different between the two subjects.

\subsection{Accommodating a Partially Known Structure and Latent Confounders.}

It is not always needed to perform a full search. The FIC algorithm is easily modified to start from a predefined structure. We specify the most complex graph that we are willing to consider, and search through subsets of this graph only. This is obtained by restricting the range of possible combinations of parents that can influence node $j$, as opposed to creating all $2^{p-1}$ combinations.

A related approach would be to make a compromise between identifying the latent data generating process and the need for graphical structures with small MSE for the focus estimators, by using as a starting point the skeleton of the graph (estimated by algorithms such as $\mathrm{HC}$ or $\mathrm{PC}$ ), which specifies some relations as implausible, diminishing the computational burden on the algorithm, and others for which the directionality exists, but is unknown. The algorithm will search for subgraphs and orientations of the edges. The estimated network starting from the HC skeleton is presented in Figure 4(d).

Extending the DAG solution to ancestral graphs, is supported by the smaller prediction errors that can be obtained. Again, both an average model, and an individual one can be estimated. Using the 'Mathematics marks' dataset, the overall AG model is presented in Figure 4(g) and the network for the top ranked student in Figure 4(h). In both cases, allowing for correlation between residuals for $A N L$ and $M E C H$ produces better FIC scores. We note that for the bottom ranked student no such couple of correlated errors proved to provide better scores.

\subsection{FIC Compared to Competing Methods for the Benchmark Datasets}

In terms of LOO-CV, see Table 3, FIC outperforms the other algorithms in the DAG class, except for the 'Mineral' dataset and it outperforms SIN in the GGM class. When compared 


\begin{tabular}{llccccc}
\hline & & Grades & Glucose & Mineral & Borings & Head \\
\hline DAG & FIC & $\mathbf{3 . 3 8}$ & $\mathbf{4 . 4 4}$ & 4.74 & 1.65 & $\mathbf{2 . 3 6}$ \\
& HC(BIC) & 3.41 & 4.46 & 4.73 & 1.66 & 2.43 \\
& HC(AIC) & 3.41 & 4.46 & $\mathbf{4 . 7 2}$ & 1.65 & 2.44 \\
& PC $(\alpha=0.1)$ & 3.40 & 4.48 & 4.77 & 1.65 & 2.93 \\
& PC $(\alpha=0.05)$ & 3.66 & 4.63 & 5.06 & 1.65 & 2.93 \\
GGM & FIC & $\mathbf{2 . 6 0}$ & $\mathbf{4 . 0 0}$ & $\mathbf{3 . 7 7}$ & 0.81 & 1.80 \\
& SIN $(\alpha=0.25)$ & 2.67 & 4.31 & 4.29 & 0.82 & 1.81 \\
& SIN $(\alpha=0.15)$ & 2.93 & 4.19 & 4.32 & 0.77 & 2.72 \\
& SIN $(\alpha=0.05)$ & 3.13 & 4.39 & 4.33 & 1.04 & 2.55 \\
& GLasso(BIC) & 2.70 & 4.05 & 3.92 & $\mathbf{0 . 6 9}$ & $\mathbf{1 . 4 9}$ \\
AG & FIC & 2.61 & - & 3.73 & 0.6 & 1.49 \\
\hline
\end{tabular}

Table 3: Five real datasets. LOO-CV averaged squared prediction errors for the estimated DAG, GGM and AG. For 'Glucose' dataset the AG is the same as the estimated DAG, and no prediction errors are presented.

to GLasso, the results are similar, except for the 'Head' dataset where the differences are larger.

On the 'Mineral' dataset, the FIC-based model has a slightly worse LOO prediction error because, even though this model performs better on 3 variables out of 6 , for the remaining variables the performance is worse than that of the $\mathrm{HC}$ solution because of possible acyclicity constraints or infeasibility conditions.

Extending towards ancestral graphs by allowing for correlated errors seems to improve the prediction performance of the estimated graphical structure except for the 'Glucose' dataset.

\section{Selection From a List of Prespecified Gaussian Graphical Models}

Assuming multivariate normality for the data, we can define focuses on the entire covariance matrix. We stress that in this part we use the FIC methodology to select among a few candidate models, the ones that perform best with respect to the estimation of specific focuses. We do not 'learn' plausible models from the data, but instead build them from theory and by making educated guesses or reasonable assumptions. Once such a list of candidate models has been constructed, the FIC methodology can aid in the selection of best fitting models (see Claeskens and Hjort, 2008a).

Using the 'Mathematics grades' dataset, six focuses have been defined: two generalized standard deviation measures $\mu_{1}=(\operatorname{det} \Sigma)^{1 / 8}$ and $\mu_{2}=\sqrt{\operatorname{tr}(\Sigma)}$, two partial regression coefficients $\mu_{3}=\beta_{S T A T ; M E C H}(S T A T \leftarrow M E C H)$ and $\mu_{4}=\beta_{A L G ; V E C T}$ (i.e. $A L G \leftarrow$ $V E C T)$, an upper diagonal average measure of correlation $\mu_{5}=(1 / 10) \Sigma_{i<j} \operatorname{corr}\left(X_{i}, X_{j}\right)$ and $\mu_{6}$ the correlation between a linear combination of MECH, VEC and ALG and ANL. The goal is to find for these particular focuses of interest, models that provide lower MSE values when a particular focus is estimated. Different from the previous application of FIC selection, we now define focuses that refer to the entire $\Sigma$ matrix, and do not concentrate 


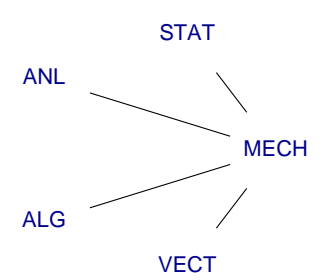

(a) Model $_{1}$

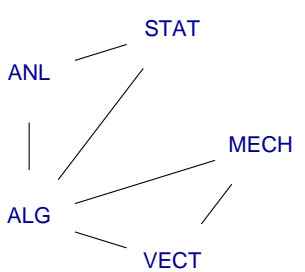

(e) Model $_{5}$

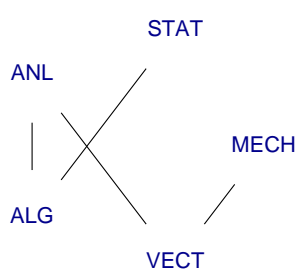

(b) $\mathrm{Model}_{2}$

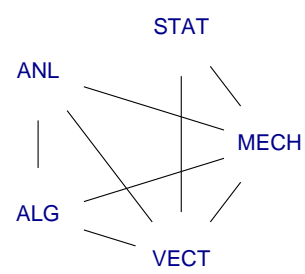

(f) Model $_{6}$

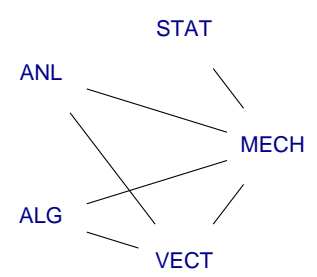

(c) $\mathrm{Model}_{3}$

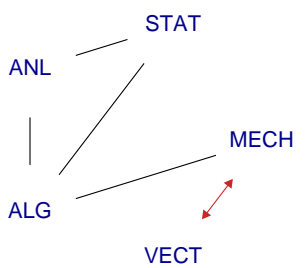

(g) $\mathrm{Model}_{7}$

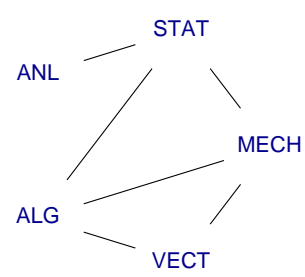

(d) $\mathrm{Model}_{4}$

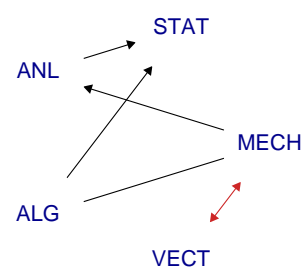

(h) $\mathrm{Model}_{8}$

Figure 5: 'Mathematics grades' dataset. 8 hypothetical candidate models are presented, from which selection of best fitting ones is intended.

on the expected value as a focal point. None of the previous search algorithms are used, as now all focuses are defined as functions of direct entries of the covariance matrix of a multivariate normal distribution. Model selection is performed by testing a sequence of models where particular elements in the concentration matrix are set to 0 , while all others are freely estimated.

Six plausible multivariate 5-dimensional normal densities with mean 0 and different covariance matrix (representing 6 different GGMs), have been proposed and the objective is to select among these models the best fitting ones in terms of estimated MSE for the six focuses. Graphically the models are presented in Figure 5. To these six models, an empty GGM as well as a fully connected one, and two particular AGs, have been added as well. The obtained results are presented in Table 4.

For this particular dataset, except for $\mu_{6}$ as focus, the estimated GGM model from Figure 4(i) was selected as the best model with respect to the MSE. For $\mu_{1}, \mu_{2}$ and $\mu_{5}$ a fully connected model comes second, and since this model estimates more parameters, it will generally provide larger variances for the focus parameter. For $\mu_{4}$ model 4 is second best, for $\mu_{6}$ the second best model is $M_{5}$. The important message is that is is possible and worthwhile to direct the model search towards finding a model with small MSE for the focus of interest. Remark too, that this second independent analysis comes to fully support the model that was estimated for different focuses and using a series of nodewise regression equations in Section 6.1.

For $\mu_{3}$, all models that do not set any edge between $S T A T$ and $M E C H$ and have thus, a corresponding 0 entry in the concentration matrix, perform equally well. They all have the same estimated bias and the same estimated variance as the null model, because with respect to $\mu_{3}$, all 5 models are equivalent. These models seem not to be heavily biased when 


\begin{tabular}{|c|c|c|c|c|c|c|c|c|c|c|c|c|}
\hline Model & \multicolumn{2}{|c|}{$\operatorname{FIC}\left(\mu_{1}\right)$} & \multirow{2}{*}{\multicolumn{2}{|c|}{ FIC $\left(\mu_{2}\right)$}} & \multicolumn{2}{|c|}{$\operatorname{FIC}\left(\mu_{3}\right)$} & \multicolumn{2}{|c|}{$\operatorname{FIC}\left(\mu_{4}\right)$} & \multicolumn{2}{|c|}{$\operatorname{FIC}\left(\mu_{5}\right)$} & \multicolumn{2}{|c|}{$\operatorname{FIC}\left(\mu_{6}\right)$} \\
\hline Empty & 91.1 & & 292.3 & & 0.2 & (1) & 38.1 & & 17.4 & & 140.5 & \\
\hline$M 1$ & 43.6 & & 139.8 & & 10.3 & & 38.1 & & 7.6 & & 91.0 & \\
\hline$M 2$ & 4.7 & & 15.1 & & 0.2 & (1) & 38.1 & & 0.9 & & 25.5 & \\
\hline M3 & 26.2 & & 84.1 & & 10.6 & & 10.8 & (3) & 4.5 & & 54.0 & \\
\hline M4 & 5.0 & & 15.9 & & 5.2 & & 9.5 & (2) & 0.9 & & 140.5 & \\
\hline M5 & 1.7 & (1) & 5.3 & (1) & 0.2 & (1) & 8.8 & (1) & 0.3 & (1) & 14.1 & (2) \\
\hline$M 6$ & 5.4 & & 17.2 & & 8.2 & & 12.9 & & 0.9 & & 20.5 & \\
\hline$M 7$ & 4.8 & & 15.3 & & 0.2 & (1) & 38.1 & & 1.0 & & 13.6 & (1) \\
\hline$M 8$ & 4.7 & (3) & 14.9 & $(3)$ & 0.2 & (1) & 38.1 & & 0.9 & (3) & 15.8 & (3) \\
\hline Full & 1.7 & (2) & 5.5 & (2) & 6.2 & & 11.8 & & 0.3 & (2) & 16.2 & \\
\hline
\end{tabular}

Table 4: Mathematics grades dataset. Focused information criterion values for six focuses and 10 possible multivariate normal models, with different covariance matrices. Models with rank (1) are preferred by the criterion.

compared to the full model and so selecting one of these models to estimate $\mu_{3}$ seems to be the proper way.

With respect to $\mu_{4}$, the first three models and the two ancestral graphs, estimate it at 0 and their scores are as well identical, but these models have large biases that dominate the estimated MSE. Models 4 and 5 are not that biased when compared to the full model, and provide also variances that are closest to the empty model. Selecting model 5 for the estimation of this particular focus is the best decision, but model 4 follows closely. For the sixth focus, the ancestral graph proposed in Figure 5(g) performs best, among all proposed models.

\section{Discussion}

Global models often can be improved for specific purposes, e.g. in terms of prediction error. We define the focused information criterion for graphical models and concentrate in this stage on small networks using directed acyclic graphs and Gaussian graphical models. The search algorithm is able to identify graphical models with better cross-validated prediction error than existing methods, such as the models identified by hill climbing, PC (with additional orientation) or the GLasso algorithm. Extensions towards larger networks are a topic of current research.

For the DAG setting, on several of the considered datasets, the FIC based method is capable of being at least as powerful in terms of leave-one-out crossvalidation error as the hill-climbing or the PC solution. On the datasets, in the GGM setting, the focused search also performed comparably or better. For the simulation settings on which we have tested the algorithm, in the large majority of cases the FIC procedure performs better.

Different models can be better in terms of MSE for particular focuses, highlighting once again that the purpose of the model which is reflected in the chosen focus to estimate, is important in the sense that different purposes, and thus different focuses, may lead to different selected models. 


\section{References}

H. Akaike. Information theory and an extension of the maximum likelihood principle. In B. Petrov and F. Csáki, editors, Second International Symposium on Information Theory, pages 267-281. Akadémiai Kiadó, Budapest, 1973.

W. Buntine. Theory refinement of Bayesian networks. In Proceedings of the 17th Conference on Uncertainty in Artificial Intelligence, pages 52-60, 1991.

G. Claeskens and N.L. Hjort. The focused information criterion. Journal of the American Statistical Association, 98:900-916, 2003. With discussion and a rejoinder by the authors.

G. Claeskens and N.L. Hjort. Model Selection and Model Averaging. Cambridge University Press, Cambridge, 2008a.

G. Claeskens and N.L. Hjort. Minimising average risk in regression models. Econometric Theory, 24:493-527, 2008b.

G.F. Cooper and E. Herskovits. A Bayesian method for the induction of probabilistic networks from data. Machine Learning, 9(4):309-348, 1992.

D. R. Cox and N. Wermuth. Multivariate dependencies: Models, Analysis and Interpretation. Chapman \& Hall, London, 1996.

A.P. Dempster. Covariance selection. Biometrics, 28(1):157-175, 1972.

D. Dor and M. Tarsi. A simple algorithm to construct a consistent extension of a partially oriented graph. Technicial Report R-185, 1992.

M. Drton and M.D. Perlman. Model selection for Gaussian concentration graphs. Biometrika, 91(3):591-602, 2004.

M. Drton and M.D. Perlman. A SINful approach to Gaussian graphical model selection. Journal of Statistical Planning and Inference, 138(4):1179-1200, 2008.

M. Drton and T.S. Richardson. Iterative conditional fitting for Gaussian ancestral graph models. In Proceedings of the 20th Conference on Uncertainty in Artificial Intelligence, pages 130-137, 2004.

D. Edwards. Introduction to Graphical Modelling. Springer, New York, 2nd edition, 2000.

J. Friedman, T. Hastie, and R. Tibshirani. Sparse inverse covariance estimation with the graphical lasso. Biostatistics, 9(3):432-441, 2008.

N.L. Hjort and G. Claeskens. Frequentist model average estimators. Journal of the American Statistical Association, 98:879-899, 2003. With discussion and a rejoinder by the authors.

N.L. Hjort and G. Claeskens. Focussed information criteria and model averaging for Cox's hazard regression model. Journal of the American Statistical Association, 101:1449-1464, 2006. 
D. Koller and N. Friedman. Probabilistic Graphical Models: Principles and Techniques. MIT Press, Cambridge, MA, 2009.

S.L. Lauritzen. Graphical Models. Oxford University Press, 1996.

K. V. Mardia, J. T. Kent, and J. M. Bibby. Multivariate Analysis. Academic Press, London, 1979 .

N. Meinshausen and P. Bühlmann. High-dimensional graphs and variable selection with the lasso. Annals of Statistics, 34(3):1436-1462, 2006.

T. Richardson and P. Spirtes. Ancestral graph Markov models. Annals of Statistics, 30(4): 962-1030, 2002.

S. Russell and P. Norvig. Artificial Intelligence. A Modern Approach. Prentice-Hall Inc, Englewood Cliffs, NJ, 1995.

G. Schwarz. Estimating the dimension of a model. Annals of Statistics, 6(2):461-464, 1978.

P. Spirtes, C. Meek, and T.S Richardson. An algorithm for causal inference in the presence of latent variables and selection bias. In C. Glymour and G. Cooper, editors, Computation, Causation and Discovery, pages 211-252. MIT Press, 1999.

P. Spirtes, C. Glymour, and R. Scheines. Causation, prediction, and search. MIT Press, Cambridge, MA, 2nd edition, 2000.

I. Tsamardinos, E. L. Brown, and C. F. Aliferis. The max-min hill-climbing Bayesian network structure learning algorithm. Machine Learning, 65(1):31 - 78, 2006.

J. Whittaker. Graphical Models in Applied Multivariate Statistics. John Wiley \& Sons, Chichester, 1990.

J. Williamson. Bayesian Nets and Causality. Philosophical and Computational Foundations. Oxford University Press, Oxford, 2005.

D. M. Witten, J. H. Friedman, and N. Simon. New insights and faster computations for the graphical lasso. Journal of Computational and Graphical Statistics, 20(4):892-900, 2011.

M. Yuan and Y. Lin. Model selection and estimation in the Gaussian graphical model. Biometrika, 94(1):19-35, 2007.

J. Zhang. On the completeness of orientation rules for causal discovery in the presence of latent confounders and selection bias. Artificial Intelligence, 172(16):1873 - 1896, 2008.

$\mathrm{X}$. Zhang and H. Liang. Focused information criterion and model averaging for generalized additive partial linear models. Annals of Statistics, 39(1):174-200, 2011. 